\title{
PRIMEROS DATOS SOBRE EL METABOLISMO DE UN ARROYO TEMPORAL MEDITERRÁNEO DE SIERRA MORENA (CÓRDOBA)
}

\author{
Salvador Mollá, Leonardo Maltchik \& Carmen Casado. \\ Dpto. Interuniversitario de Ecología de Madrid, Universidad Autónoma de Madrid \\ Ciudad Universitaria Cantoblanco. 28049 MADRID.
}

Palabras clave: Metabolismo, Producción primaria, Respiración del ecosistema, Arroyo temporal. Keywords: Metabolism, Primary production, Ecosystem respiration, Temporary stream.

\begin{abstract}
FIRST DATA ON METABOLISM OF A MEDITERRANEAN TEMPORARY STREAM IN SIERRA MORENA (CóRDOBA)

Ecosystem metabolism of Arroyo de la Montesina was studied from January to June 1992, using the Odum's diel oxygen curve method, in different periods of the hydrological cycle. This second order stream ecosystem is characterized by spatial and temporal heterogeneity. It posesses runs and pools alternately, as well as two clearly different hydrological phases: one in winter, when water flows in the channel, and another in summer, when surface water is only present in isolated pools.

Results show the relatively high productivity of this ecosystem compared to other stream ecosystems, and a gradual rise of productivity from January to June. During this period the ecosystem shifted from heterotrophy to autotrophy. This shift is related to variation in stream hydrology, i. e., the heterotrophic phase coincides with flowing water, and the autotrophic one with pools. The lack of data concerning metabolism of Mediíesranean temporary streams does not allow comparisons with similar ecosystems nor to establish general functional models. It is therefore suggested that studies of this kind be encouraged, in order to gain a better understanding of the functional processes of temporary streams in Mediterranean area
\end{abstract}

\section{INTRODUCCIÓN}

La mayoría de los estudios sobre funcionamiento de ríos se han desarrollado en sistemas fluviales de las regiones templadas, con flujo de agua permanente y bastante poco alterados (BENKE et al., 1988). A partir de estas investigaciones se han realizado generalizaciones y formulado teorías integradoras, como es el caso de 'River Continuum Concept' (VANNOTE et al., 1980), cuya validez en ríos de otras zonas, con características ambientales diferentes, ha sido puesta en duda por diversos autores (WINTERBOURN et al., 1981, 1988; BOULTON \& LAKE, 1988; RYDER \& SCOTT, 1988; entre otros).

En este contexto, apenas existen estudios que traten de forma integrada los aspectos funcionales de los ríos de las regiones áridas y semiáridas de nuestro planeta (VIDALABARCA et al., 1990: VIDAL-ABARCA et al., 1992a).
Tan sólo se han realizado trabajos de relevancia en un arroyo del desierto de Arizona (BUSH \& FISHER, 1981; FISHER et al., 1982; FISHER \& GRAY, 1983; GRIMM, 1987; FISHER \& GRIMM, 1988; GRIMM et al., 1991; entre otros), y en algunos ríos temporales de Australia (p. ej. ROUNICK et al., 1982; ROUNICK \& WINTERBOURN, 1986; COLLIER \& WINTERBOURN, 1987). El volúmen de estas investigaciones no es comparable al de los trabajos desarrollados en los sistemas fluviales de las regiones templadas. A modo de ejemplo se puede señalar que en una revisión realizada sobre las tasas metabólicas registradas en sistemas lóticos de los Estados Unidos (BOTT et al. 1985), de las casi 80 medidas presentadas, sólo 5 pertenecen a ambientes áridos, y tan sólo 2 de ellas son de zonas áridas cálidas (las otras tres corresponden a desiertos fríos).

Desde el punto de vista de la dinámica funcional, los 
ríos temporales de la zona mediterránea presentan modelos de distribución temporal del flujo de agua relacionados, principalmente, con la irregularidad del régimen de precipitaciones (VIDAL-ABARCA, 1990). Este hecho, unido a las altas temperaturas que se alcanzan durante el verano, da lugar a un ciclo hidrológico caracterizado por la irregularidad de los caudales, con crecidas de diversa magnitud y fuertes estiajes (GIUDICELLI et al., 1985), lo que sin duda ha de influir en la dinámica funcional del ecosistema (VIDAL-ABARCA et al., 1992b; SABATER et al., 1993).

El objetivo de este trabajo es realizar una primera aproximación al funcionamiento de un arroyo temporal de la zona mediterránea, empleando el metabolismo global del ecosistema como un indicador de la eficacia del sistema en el procesamiento de los recursos energéticos. El estudio recoge dos fases distintas del ciclo hidrológico (agua corriente y estiaje), con el propósito de determinar qué procesos tróficos (autotróficos o heterotróficos) son predominantes en cada una de ellas.

\section{ÁREA DE ESTUDIO: EL ARROYO DE LA MONTESINA.}

El arroyo de la Montesina se encuentra situado en el extremo Oeste de la provincia de Córdoba, en la región de Sierra Morena (fig. 1). Es un tributario del río Bembézar, perteneciente a la cuenca del Guadalquivir. Tiene una longitud de casi $29 \mathrm{Km}$ desde su origen a $680 \mathrm{~m}$ sobre el nivel del mar, hasta su confluencia con el río Bembézar, a una altitud de $205 \mathrm{~m}$, y drena una superficie de 128.25 $\mathrm{Km}^{2}$.

Sus aguas fluyen de Norte a Sur sobre un basamento silíceo impermeable compuesto principalmente por rocas ígneas y metamórficas, discurriendo por un estrecho valle que se ensancha en algunos tramos. El lecho del arroyo se encuentra integrado, generalmente, por cantos rodados de distinta granulometría, alternando con arenas y limos en los tramos más abiertos y de corriente más lenta.

El área tiene una precipitación media anual de 527.1 $\mathrm{mm}$, y una temperatura media anual de 16.1 "C. La irregular distribución de las precipitaciones determina el marcado carácter temporal del arroyo. Las lluvias se inician débilmente en Septiembre, alcanzando su máxima intensidad entre Octubre y Febrero. A partir de Marzo la precipitación disminuye gradualmente hasta el verano, cuando las lluvias son prácticamente nulas, exceptuando alguna precipatación veraniega de carácter tormentoso (ENRESA,

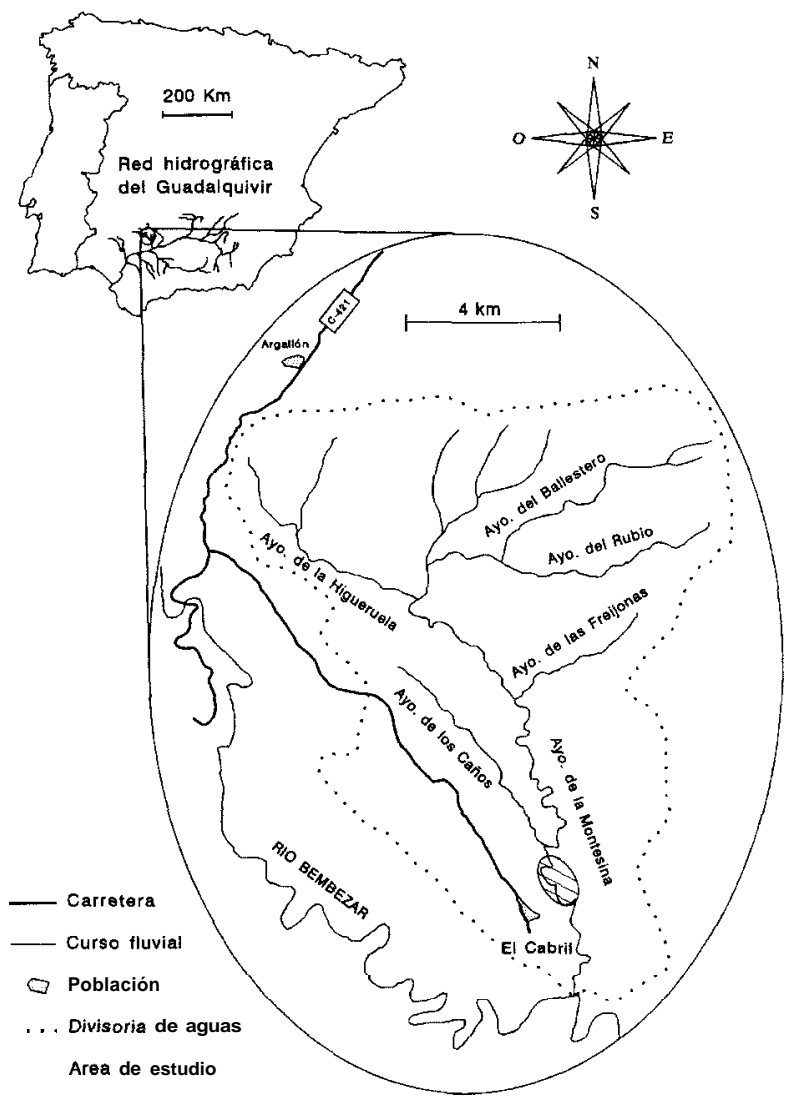

FIGURA 1.- Mapa de situación del área de estudio. El área rayada señala el segmento fluvial estudiado.

FIGURE 1.- Location of study site. Striped area indicates the stream reach studied.

1990).

Sólo en el invierno el cauce mantiene un flujo continuo, el cual disminuye progresivamente hasta el verano. En Julio ya no existe agua corriente pero se mantienen algunas pozas, las cuales perduran hasta las lluvias de otoño que restauran las aguas corrientes en el cauce. Este patrón se interrumpe a menudo con la llegada de lluvias torrenciales y consecuentes avenidas.

La zona norte de la cuenca del Montesina presenta grandes extensiones de encinar adehesado, que alternan con cultivos extensivos de olivar. El resto de la cuenca se encuentra dominado por el bosque xeromediterráneo y sus etapas de sustitución, con un estrato arbóreo integrado principalmente por encinas (Quercus rorundifolia Lam.). La parte más meridional de la cuenca del Montesina, y al sur de ésta, es una de las pocas zonas de la provincia donde todavía es importante la presencia de alcornocales 

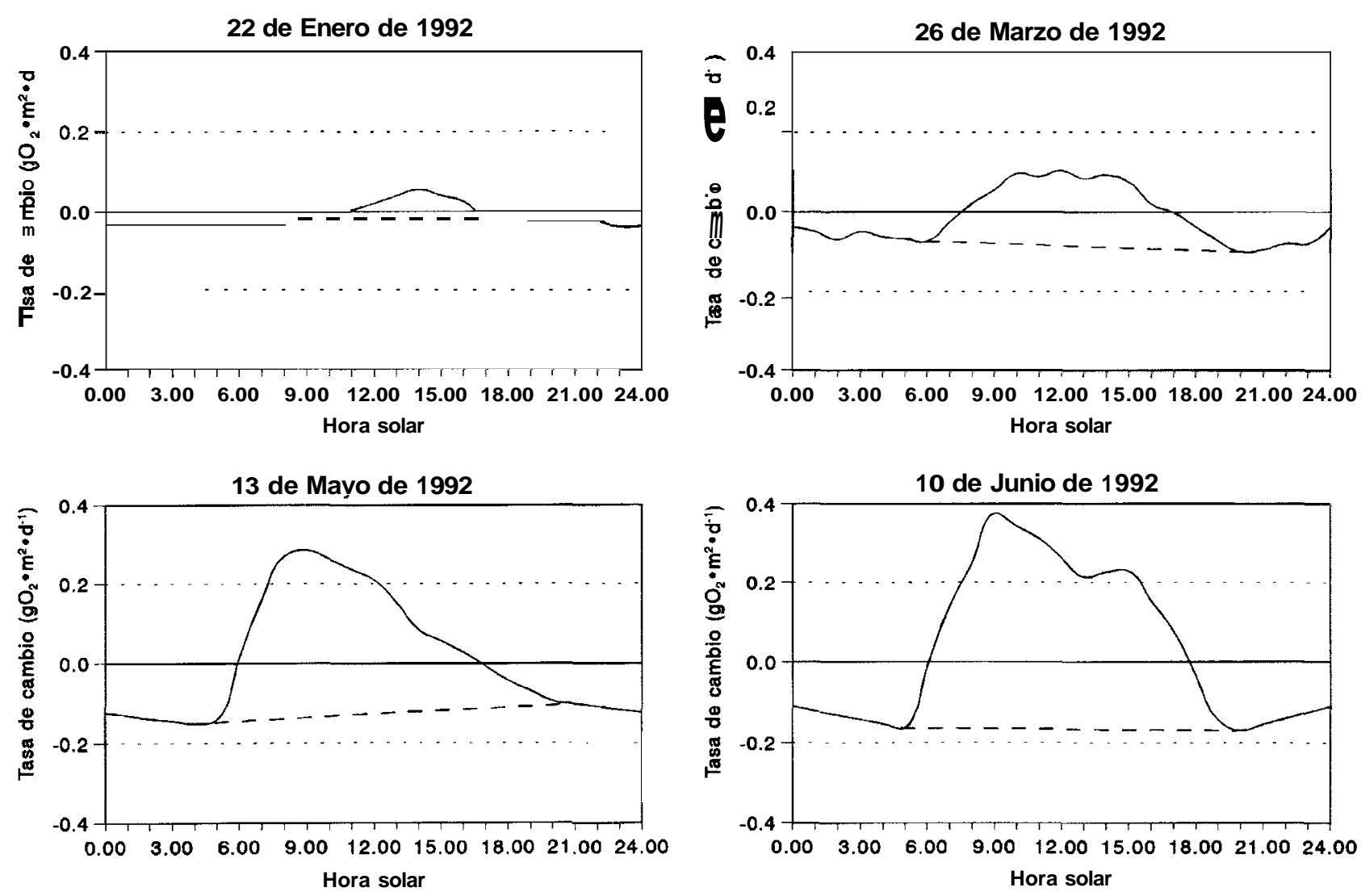

FIGURA 2.- Curvas de tasa de cambio de oxígeno, corregidas para la difusión, obtenidas en las distintas campañas de muestreo. La línea discontinua representa la estima de la respiración diurna.

FIGURE 2.- Oxygen rate of change curves on the different sampling dates. Solid line represents the rate of change curve corrected for diffusion, and dashed line represents the estimate of daytime respiration.

(Quercus suber L.) acompañando a los encinares. Esta vegetación se encuentra alterada por grandes repoblaciones de pino piñonero (Pinus pinea L.) y marítimo (Pinus pinaster Aiton), que en general no prosperan con facilidad. También se han realizado repoblaciones con eucalipto (Eucalyptus spp), aunque ocupan extensiones muy inferiores, limitándose a pequeñas manchas en el paisaje. Las riberas de esta parte de Sierra Morena presentan algunos sotos constituidos principalmente por álamos (Populus spp), sauces (Salix spp), olmos (Ulmus spp), y alisos (Alnus spp), aunque en arroyos de poca importancia, como el arroyo de la Montesina, es más habitual la presencia de tarais (Tamarix spp), adelfas (Nerium oleander L), sauzgatillos (Vitex agnus-castus L.), lentiscos (Pistacia lentiscus L.), y zarzas (Rubus spp) (ENRESA 1990).

En el tramo seleccionado para la realización del estudio (fig. 1) existe una abundante vegetación sumergida integrada por Chara vulgaris L, Chara globularis Thuill, Ca- llitriche stagnalis Scop, Ranunculus peltatus subsp peltatus Schrank y Nasturtium officinale $\mathrm{R}$. Br.

\section{METODOLOGÍA}

La zona de muestreo se encuentra situada en la parte meridional de la cuenca del Montesina, por debajo de la confluencia con el arroyo de los Caños (fig. 1). Las medidas se realizaron en los meses de Enero, Marzo, Mayo y Junio de 1992, durante días de cielo despejado.

La tasa de producción primaria neta (PPN) y la respiración nocturna $\left(\mathrm{R}_{\mathrm{n}}\right)$ se han calculado mediante la técnica simple de la curva diaria de oxígeno (ODUM, 1956), basada en el cambio de la concentración de oxígeno disuelto durante un período de 24 horas. Este método es aplicable cuando las curvas diarias de oxígeno obtenidas por la técnica de las dos estaciones son similares, como ocurría en nuestro caso. La difusión se ha determinado mediante el 
análisis de la curva de tasa de cambio nocturna (ODUM, 1956; McDIFFETT et al., 1972; OWENS, 1974).

La medida de la respiración diurna real es imposible mediante este método (HALL \& MOLL, 1975). A fín de solventar este problema, se asume que una línea trazada en la curva de tasa de cambio desde el punto más bajo antes del amanecer hasta el mínimo después del anochecer (fig. 2), proporciona una buena aproximación de la respiración diurna (ODUM \& WILSON, 1962; KELLY et al.. 1974; HALL \& MOLL, 1975). Las estimas de la respiración diurna $(\mathrm{R}$,$) se emplearon para calcular la producción$ primaria bruta $(\mathrm{PPB})$ y la respiración diaria $\left(\mathrm{R}_{24}\right)$ del arroyo de la Montesina.

En cada ocasión, el oxígeno disuelto y la temperatura del agua se registraron durante al menos 24 horas. Cuando había agua corriente (Enero y Marzo), las medidas de oxígeno disuelto y temperatura se realizaron con un oxímetro WTW-196. El pH se midió con un pH-metro WTW-96, y la radiación fotosintéticamente activa (RFA, longitud de onda: 400-700 nm) con un sensor quantum LI-Cor 190SA. Estos instrumentos se encontraban conectados a un almacenador de datos LI-1000 que grababa la media de los datos cada 15 minutos. Las medidas de oxígeno disuelto re- gistradas por el oxímetro se calibraron cada seis horas con muestras de agua analizadas mediante el método Winkler (APHA, 1989).

En Mayo y Junio, cuando no había agua corriente superficial, las medidas se realizaron en pozas próximas a los puntos de muestreo de Enero y Marzo. Debido a que el oxímetro no puede registrar medidas sin una mínima velocidad de la corriente, y no se disponía de agitadores para los electrodos, el oxígeno disuelto en el agua se analizó mediante el método Winkler, a partir de muestras de agua tomadas cada 3 horas.

El índice $\mathrm{P} / \mathrm{R}$ se calculó como la razón entre la producción primaria bruta $(\mathrm{PPB})$ y la respiración diaria $\left(\mathrm{R}_{24}\right)$. El metabolismo neto diario (MND) se calculó como la diferencia entre la producción primaria neta y la respiración nocturna $\left(M N D=P P N-R_{n}\right)$. Este índice $(M N D)$ es semejante al $\mathrm{P} / \mathrm{R}$, proporcionando información sobre la dominancia de los procesos autotróficos o heterotróficos en el tramo de estudio, expresándose en gramos de oxígeno por metro cuadrado y día (BOTT et al., 1978; BOTT, 1983; MINSHALL et al., 1983).

Complementariamente se realizaron medidas puntuales de la conductividad de las aguas (conductivímetro WTW

TABLA 1.- Valores de las tasas metabólicas del arroyo de la Montesina y principales parametros físico-químicos relacionados con la producción TABLE 1.- Metabolic rates recorded in Arroyo de la Montesina, and main physical and chemical parameters related to production.

\begin{tabular}{|c|c|c|c|c|}
\hline Variable & Enero & Marzo & Mayo & Junio \\
\hline $\mathrm{T}^{\mathrm{a}}$ media del agua $\left({ }^{\circ} \mathrm{C}\right)$ & 4.9 & 10.8 & 21.3 & 22.7 \\
\hline pH (rango) & $8.0-8.4$ & $7.8-8.3$ & $7.2-7.6$ & $7.8-8.8$ \\
\hline$\left[\mathrm{O}_{2}\right]$ disuelto $(\mathrm{ppm})($ rango $)$ & $11.1-12.9$ & $9.4-11.6$ & $6.8-16.1$ & $6.9-24.1$ \\
\hline \% saturación $\mathrm{O}_{2}$ (rango) & $88-111$ & $80-123$ & $74-202$ & $61-331$ \\
\hline Conductividad ( $\mu \mathrm{S} \mathrm{cm-')}$ & 450 & 632 & 597 & 595 \\
\hline Caudal $\left(1 \mathrm{~s}^{-1}\right)$ & 19.7 & 5.2 & - & - \\
\hline Velocidad del agua $\left(\mathrm{cm} \mathrm{s}^{-1}\right)$ & 2.9 & 2.7 & 0 & 0 \\
\hline Profundidad media (m) & 0.20 & 0.18 & 0.17 & 0.12 \\
\hline R.F.A. (Em-2 día-') & 4.878 & 25.567 & 41.729 & 58.483 \\
\hline$f\left(\mathrm{~m} \mathrm{~h}^{-1}\right)$ & 0.035 & 0.035 & 0.007 & 0.008 \\
\hline PPB (g O, m² día-') & 0.451 & 1.624 & 3.408 & 4.722 \\
\hline $\mathrm{R}, \quad\left(\mathrm{g} \mathrm{O}, \mathrm{m}^{-2}\right.$ día-' $)$ & 0.812 & 1.919 & 3.071 & 3.742 \\
\hline M.N.D. $\left(\mathrm{g} \mathrm{O}_{2} \mathrm{~m}^{-2}\right.$ día $\left.^{\mathrm{l}}\right)$ & -0.361 & -0.295 & 0.403 & 0.980 \\
\hline $\mathrm{P} / \mathrm{R}$ & 0.555 & 0.846 & 1.134 & 1.262 \\
\hline
\end{tabular}

R.F.A..- Radiación Fotosintéticamente Activa; PPB.- Producción Primaria Bruta

$\mathrm{R}_{24}$-- Respiración Diaria; P/R.- PPB/R ${ }_{24}$; M.N.D.- Metabolismo Neto Diario

f.- Coeficiente de intercambio de oxígeno. 
LF-96). Asimismo se midieron la velocidad de la corricnte (correntímetro Global Water), y el área transversal del cauce inundado en tres transectos de la estación de muestreo, con objeto de calcular el caudal circulante.

\section{RESULTADOS}

La tabla 1 muestra las tasas metabólicas calculadas a partir de las medidas realizadas, además de algunas variables físico-químicas de interés. A nivel general, hay que señalar la importante reducción de caudal que se produce entre Enero y Marzo, así como la ausencia de agua corriente en Mayo y Junio.

El metabolismo del arroyo de la Montesina muestra un incremento gradual de la producción primaria bruta (PPB) y de la respiración de las comunidades $\left(\mathrm{R}_{24}\right)$ desde Enero hasta Junio. Se aprecia, sin embargo, que la primera (PPB) sufre un aumento mayor que la segunda $\left(\mathrm{R}_{24}\right)$, sobrepasándola a partir de Mayo. Como consecuencia de ello, el índice $\mathrm{P} / \mathrm{R}$ es mayor que 1 a partir de Mayo, y el MND adquiere valor positivo, indicando el predominio de los procesos autotróficos. En la tabla 1 observamos que este cambio coincide con la desaparición del flujo superficial, es decir con la época en la que el arroyo se muestra como un rosario de pozas sin comunicación superficial, y que se corresponde con un incremento de la radiación (RFA) y de la temperatura del agua. La primera (RFA) aumenta tanto en su intensidad, como en su duración, al hacerse los días más largos conforme se aproxima el solsticio de verano (fig 3).

Un hecho destacable de la tabla 1 es el rango de varia-

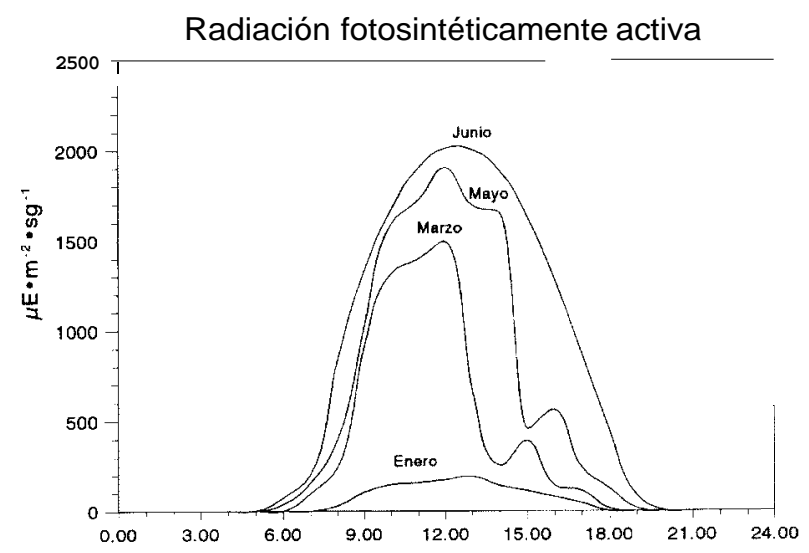

FIGURA 3.- Variación diaria de la radiación fotosintéticamente activa en los cuatro muestreos realizados.

FIGURE 3.- Variation of photosynthetically active radiation over 24 hours on the four sampling dates. ción que presentan el pH y, sobre todo, la concentración de oxígeno disuelto. En el mes de Junio, la concentración de oxígeno disuelto llega a oscilar entre poco más del $60 \%$ de saturación hasta por encima del $300 \%$. Esta variación se explica por la alta actividad metabólica del sistema, como muestran las tasas de producción primaria bruta (PPB) y respiración $\left(R_{24}\right)$.

Por otra parte, el coeficiente de intercambio de oxígeno (f), permanece prácticamente constante en los muestreos con agua corriente $\left(0.035 \mathrm{~m} \mathrm{~h}^{-1}\right)$ y con agua estacada (0.007-0.008 $\mathrm{m} \mathrm{h}^{-1}$ ), presentando una gran diferecia entre ambos períodos.

\section{DISCUSIÓN}

En el arroyo de la Montesina, la producción primaria bruta (PPB) aumenta a lo largo del período hidrológico conforme lo hacen la radiación fotosintéticamente activa y la temperatura del agua. La respiración de la comunidad $\left(\mathrm{R}_{24}\right)$ sigue este mismo patrón de variación, tendiendo a ser proporcionalmente inferior entre los equinoccíos de primavera y otoño. Dentro de estas tendencias, la actividad metabólica del sistema aumenta de forma más notable cuando no existe corricnte dc agua superficial. La importancia de la variación estacional de radiación solar y del régimen hidrológico sobre las tasas metabólicas del sistema se ha observado en otros otros arroyos someros, donde la luz puede alcanzar la superficie del agua sin ser tamizada por la vegetación de ribera (NAIMAN \& SEDELL, 1980; BOTT. 1983).

Como consecuencia de la variación estacional de la producción primaria bruta y de la respiración, el metabolismo neto diario (MND) y el cociente $\mathrm{P} / \mathrm{R}$ dan valores que indican heterotrofía del sistema (en el sentido de productor o consumidor neto de materia orgánica) sólo en los meses de invierno (Enero y Marzo).

En relación con estos índices (MND y P/R) se deben hacer algunas puntualizaciones. Las metodologías basadas en la variación de la concentración de gases disueltos, como la empleada en este trabajo, generan estimas de la producción primaria en base al metabolismo global del ecosistema, lo que comprende fotosíntesis y respiración de las comunidades acuáticas. En estos métodos, la estima de la producción primaria bruta ( $\mathrm{PPB}$ ) es siempre imprecisa, debido a que está basada, a su vez, en estimas de la respiración diurna a partir de la respiración nocturna. Este hecho afecta directamente al índice $P / R$, que es el cociente entre las estimaciones de la producción primaria bruta $(\mathrm{PPB})$ y 
la respiración de las comunidades del sistema $\left(\mathrm{R}_{24}\right)$. Por ello se puede considerar que el metabolismo neto diario (MND) es un mejor indicador de los procesos metabólicos del ecosistema, ya que se obtiene directamente a partir de la curva de tasa de cambio de oxígeno (BOTT et al.. 1978; BOTT, 1983).

Es importante señalar que estos índices ( $P / R$ y $M N D)$ son, efectivamente, indicadores de la autotrofía y heterotrofía del sistema en el sentido de productor o consumidor neto de materia orgánica, pero no son adecuados para valorar la importancia relativa de la materia orgánica autóctona y alóctona en el mantenimiento de las comunidades de consumidores (ROSENFELD \& MACKEY 1987; MEYER 1989).

La tabla 2 recoge algunas de las tasas metabólicas obte- nidas en distintos sistemas fluviales. Todos los datos de esta tabla se obtuvieron con métodos basados en el cambio de la concentración de oxígeno disuelto en el agua del sistema acuático.

Los valores estimados de la PPB y la $\mathrm{R}_{24}$ en el arroyo de la Montesina se pueden considerar intermedios en relación con las tasas de producción dc otros ecosistemas fluviales de la Tierra (tabla 2). Sin embargo, hemos de tener en cuenta que estos resultados pueden estar subestimados debido a que, como consecuencia de la sobre-saturación de las aguas del Montesina en algunos momentos (ver tabla 1), parte del oxígeno producido por fotosíntesis puede ser liberado en forma de burbujas, sin ser entonces detectado como oxígeno disuelto en el agua.

Una revisión general de los datos de la tabla 2 pone de

TABLA 2.- Tasas metabólicas de algunos ecosistemas fluviales expresadas en $\mathrm{g} \mathrm{O}_{2} \mathrm{~m}^{-2} \mathrm{dia}^{-1}$.

TABLE 2.- Metabolic rates for selected stream ecosystems expressed in $\mathrm{g} \mathrm{O}_{2} \mathrm{~m}^{-2}$ day $^{-1}$.

\begin{tabular}{|c|c|c|c|c|c|}
\hline $\begin{array}{l}\text { Bioma (ODUM 1971) } \\
\text { y Nombre del Río }\end{array}$ & РPB & $\mathbf{R}_{24}$ & $\mathrm{P} / \mathrm{R}$ & Periodo & Referencia \\
\hline \multicolumn{6}{|l|}{ DESIERTO } \\
\hline Sycamore Creek & 9.85 & 10.50 & 0.94 & Agosto & GRIMM \& FISHER 1984 \\
\hline Pinto Creek (Est 2) & 5.30 & 4.30 & 1.23 & Jun & LEWIS \& GERKING 1979 \\
\hline Pinto Creek (Est 1) & 3.45 & 3.84 & 0.90 & Jun & LEWIS \& GERKING 1979 \\
\hline \multicolumn{6}{|l|}{ CHAPARRAL } \\
\hline Ayo. Montesina & 2.30 & 2.23 & 1.03 & Ene-Jul & Este trabajo \\
\hline \multicolumn{6}{|l|}{ PRADERA TEMPLADA } \\
\hline Big Creek & 9.19 & 17.30 & 0.52 & Jun-Oct & BACHMANN et al. 1988 \\
\hline
\end{tabular}

\begin{tabular}{|c|c|c|c|c|c|}
\hline \multicolumn{6}{|l|}{ Sureste de EEUU } \\
\hline Rapphannoch River & 6.10 & 7.30 & 0.84 & May-Jun & HORNBERGER et al. 1977 \\
\hline S. Fork Rivanna R. & 2.10 & 3.40 & 0.62 & May-Jul & HORNBERGER et al. 1977 \\
\hline Rivanna River & 2.10 & 5.10 & 0.41 & Jul-Set & HORNBERGER et al. 1977 \\
\hline South River & 2.00 & 5.30 & 0.38 & Ago-Set & HORNBERGER et al. 1977 \\
\hline Mechums River & 1.50 & 2.90 & 0.52 & Abr-Ago & HORNBERGER et al. 1977 \\
\hline New Hope Creek & 0.80 & 1.30 & 0.62 & Media anual & HALL 1972 \\
\hline Black Creek & 0.80 & 4.10 & 0.20 & Media anual & MEYER \& EDWARDS 1990 \\
\hline \multicolumn{6}{|l|}{ Centro Este de EEUU } \\
\hline Buffalo Creek & 5.62 & 2.16 & 2.60 & Agosto & McDIFFET et al. 1972 \\
\hline \multicolumn{6}{|l|}{ Noreste de EEUU } \\
\hline Fort River & 1.80 & 3.70 & 0.49 & Media anual & FISHER\&CARPENTER 1976 \\
\hline Baker River & 0.40 & 1.90 & 0.21 & Jul-Ago & HORNBERGER et al. 1977 \\
\hline
\end{tabular}


manifiesto la gran heterogenidad existente. El metabolismo de un río o arroyo depende de una amplio número de factores como la radiación incidente, la disponibilidad de nutrientes, la temperatura del agua, el régimen hidrológico, la morfología del cauce, etc. cuya importancia relativa depende a, su vez, de la que tengan el resto de los factores (BOTT, 1983).

En general se considera que los arroyos de cabecera (primer y segundo orden) son ríos heterotróficos, en los que la producción autóctona es complementada por aportes de materia orgánica alóctona procedente de los ecosistemas terrestres (HYNES, 1963; PETERSEN \& CUMMINS, 1974; CUMMINS, 1974). Esta idea se basa en el extenso conocimiento que se tiene de los ríos, generalmente permanentes, de cuencas forestadas de la zona templada, dándose por aceptado que sólo en ríos de cuencas intervenidas por el hombre, donde se producen entradas no naturales de nutrientes, se pueden presentar casos de ecosistemas fluviales cuyo soporte energético sea la producción autóctona. MINSHALL (1978) discutió esta generalización señalando la escasez de estudios sobre ríos de otras regiones, apuntando que en las zonas bioclimáticas donde los arroyos de cabecera no sufren el tamizado de la luz por parte de bosques espesos (praderas, zonas áridas y semiáridas), la producción autóctona puede representar una parte muy significativa de los recursos energéticos del sistema.

En la tabla 2 observamos que en los dos primeros biomas de la tabla, los desiertos y chaparrales, se registran tasas de producción primaria, relativamente altas, muy próximas o superiores a las tasas de respiración. Las praderas presentan una posición intermedia, con altas tasas de producción y muy altas tasas de respiración. Por último. los sistemas de los bosques templados caducifolios y bosques de lluvia tienen, por término medio, las producciones más bajas. Estas tendencias se ven modificadas, en muchos casos, por la intervención humana, de forma que los ríos de zonas agrícolas suelen presentar una alta producción primaria a causa de la destrucción de sus riberas, y el aporte extraordinario de nutrientes, procedentes de la fertilización de los campos (p. ej. BACHMANN et al., 1988; McDIFFETT et al., 1972). En otros casos, la existencia de características naturales extraordinarias modifica esta clasificación por biomas. Por ejemplo Silver Springs, originado a partir de fuentes termales, presenta tasas de producción de hasta $35 \mathrm{gO}_{2} \mathrm{~m}^{2} \mathrm{~d}^{-1}$ en primavera (ODUM, 1956), presentando una tasa de producción mínima de 13 $\mathrm{gO}_{2} \mathrm{~m}^{2} \mathrm{~d}^{-1}$ durante un ciclo anual (KNIGHT, 1983).
En nuestro caso nos encontramos con un arroyo que en la mayoría de los períodos estudiados presenta tendencia a la autotrofía. La producción primaria, así como la respiración, son bajas cuando el agua fluye y altas cuando no existe corriente de agua superficial.

Debido a que los datos aportados sólo comprenden seis meses no es posible establecer conclusiones definitivas, ni saber si el arroyo de la Montesina es netamente heterotrófico o autotrófico cn un ciclo anual. Los resultados obtenidos parecen indicar que en el arroyo de la Montesina se pueden diferenciar dos fases con funcionamientos distintos, relacionadas con la variación hidrológica del sistema: 1) Fase de agua corriente, con tasas de producción bajas y predominancia de los procesos heterotróficos; y 2) Fase de agua estancada, en la que no existe flujo superficial sino un rosario de "charcas" aisladas, con tasas de producción moderadamente altas y predominancia de los procesos autotróficos.

Estudios a más largo plazo permitirán comprobar si la autotrofía en el arroyo de la Montesina responde a la situación hidrológica (agua corriente o pozas), o sólo depende de factores como la radiación y la temperatura del agua.

La realización de otros estudios sobre el metabolismo global de los ríos y arroyos de la región mediterránea permitirían profundizar en el conocimiento de los ecosistemas fluviales de nuestro territorio, establecer comparaciones y elaborar modelos generales sobre su funcionamiento.

\section{AGRADECIMIENTOS}

Este trabajo se ha realizado con cargo al proyecto "Estructura y funcionamiento de los ecosistemas acuáticos del área de 'El Cabril' (Córdoba)", financiado por INI Medioambiente y la Empresa Nacional de Residuos Radiactivos, S.A. (ENRESA).

\section{BIBLIOGRAFIA}

APHA, AWWA, WPCF. 1989. Standard methods for the examination of water and waste water. 17th edition. American Public Health Association. Washington DC.

BACHMANN, R.W., K.J. KORTGE \& T.E. ROBERTSON, 1988. Primary production in a small agricultura1 stream. Verh. Internat. Verein. Limnol., 23: 1179-1182.

BENKE, A.C., C.A.S. HALL, C.P. HAWKINS, R.H. LOWE-MCCONNEL, J.A. STANFORD, K. SURER- 
KROPP \& J.V. WARD, 1988. Bioenergetic considerations in the analisys of stream ecosystems. J. N. Am. Benthol. Soc., 7: 480-502.

BOTT. T. L., 1983. Primary productivity in streams. In: Stream Ecology. Application and testing of general ecological theory. (J.R. BARNES \& G.W. MINSHALL, eds.): pp. 29-53. Plenum Press. New York.

BOTT, T.L., J.T. BROCK, C.E. CUSHING, S.V. GREGORY, D. KING \& R.C. PETERSEN, 1978. A comparison of methods for measuring primary productivity and community respiration in streams. Hydrobiologia, 60: 312.

BOTT, T.L., J.T. BROCK, C.S. DUNN, R.J. NAIMAN, R.W. OVINK \& R.C. PETERSEN. 1985. Benthic community metabolism in four temperate stream systems: An interbiome comparison and evaluation of the river continuum concept. Hydrohiologia, 123: 3-45.

BOULTON, A. \& P.S. LAKE, 1988. Australian temporary streams- some ecological characteristics. Verh. Internat. Verein. Limnol., 23: 1380-1383.

BUSH, D.E. \& S.G. FISHER, 1981. Metabolism of a desert stream. Freshw. Biol., 11: 301-307.

COLLIER, K.J. \& M.J. WINTERBOURN, 1987. Faunal and chemical dynamics of some acid and alkaline New Zealand streams. Freshw. Biol., 18: 227-240.

CUMMINS, K.W., 1974. Structure and function of stream ecosystems. BioScience, 24: 631-641.

ENRESA, 1990. Proyecto de $I+D$ sobre el funcionamiento de los ecosistemas del área de Sierra Alharrana (Córdoba)-Documentación Básica. ENRESA. Madrid.

FISHER, S.G. \& S.R. CARPENTER, 1976. Ecosystem and macrophyte primary production of the Fort River, Massachusetts. Hydrohiologia, 49: 175-187.

FISHER, S.G., L.J. GRAY, N.B. GRIMM \& D.E. BUSH, 1982. Temporal succesion in a desert stream ecosystem following flash flooding. Ecol. Monogr., 52: 93-110.

FISHER, S.G. \& L.J. GRAY, 1983. Secondary production and organic matter processing by collector macroinvertebrates in a desert stream. Ecology, 64 (5): 1217-1224.

FISHER, S.G. \& N.B. GRIMM, 1988. Disturbance as a determinant of structure in a Sonoran Desert stream ecosystem. Verh. Internat. Verein. Limnol., 23: 11831189.

GIUDICELLI, J., M. DAKKI \& A. DIA, 1985. Caractéristiques abiotiques et hydrobiologiques des eaux courantes méditerranéennes. Verh. Internat. Verein. Limnol., 22: 2094-2101.
GRIMM, N.B., 1987. Nitrogen dynamics during succession in a desert stream. Ecology, 68: 1157-1170.

GRIMM, N.B. \& S.G. FISHER, 1984. Exchange between interstitial and surface water: implications for stream metabolism and nutrient cycling. Hydrohiologia, 111 : 219-228.

GRIMM, N.B., H.M. VALLET, E.H. STANLEY \& S.G. FISHER, 1991. Contribution of the hyporheic zone to stability of an arid-land stream. Verh. Internat. Verein. Limnol., 24: 1595-1599.

HALL, C.A.S, 1972. Migration and metabolism in a temperate stream ecosystem. Ecology, 53: 585-604.

HALL, C.A.S. \& R. MOLL, 1975. Methods of assessing aquatic primary productivity. In Primary productivity of the Biosphere (H. LIETH \& R.H. WHITTAKER, eds): pp. 19-53. Springer-Verlag. Berlin.

HORNBERGER, G.M., M.G. KELLY \& B.J. COSBY, 1977. Evaluating eutrophication potential from river community productivity. Wat. Res. Bull.. 11: 65-69.

HYNES, H.B.N., 1963. Imported organic matter and secondary production in streams. Proceedings of the XVI International Congress of Zoology, 4: 324-329.

KELLY, M.G., G.M. HORNBERGER \& B.J. COSBY, 1974. Continuous automated measurement of rates of photosynthesis and respiration in an undisturbed river community. Limnol. Oceanogr., 19: 305-312.

KNIGHT, R.L.. 1983. Energy basis of ecosystem control at Silver Springs, Florida. In: Dynamics of lotic ecosystems (T. D. FONTAINE \& S. M. BARTELL, eds.): pp. 161-179. Ann Arbor Scien. Publ. Ann Arbor, Michigan.

LEWIS, M.A. \& S.D. GERKING, 1979. Primary productivity in a polluted intermitent desert stream. Am. Midl. Nat., 102: 172-174.

McDIFFETT, W.F., A.E. CARR \& D.L. YOUNG, 1972. An estimate of primary productivity in a Pennsylvania trout stream using a diurnal oxygen curve technique. Am. Midl. Nat., 87: 564-570.

MEYER, J.L., 1989. Can P/R ratio be used to asses the food base of stream ecosystems? A comment on Rosenfeld \& Mackay 1987. Oikos, 54: 119-121.

MEYER, J.L. \& R.T. EDWARDS, 1990. Ecosystems metabolism and turnover of organic carbon along a blackwater river continuum. Ecology, 7 (2): 668-687.

MINSHALL, G.W., 1978. Autotrophy in stream ecosystems. BioScience, 28: 767-771

MINSHALL, G.W., R.C. PETERSEN, K.W. CUMMINS, T.L. BOTT, R.J. SEDELL, C.E. CUSHING, \& 
R.L. VANNOTE. 1983. Interbiome comparison of stream ecosystem dynamics. Ecol. Monogr., 53 (1): 1-25.

NAIMAN, R.J. \& J.R. SEDELL, 1980. Relationships between metabolic parameters and stream order in Oregon. Can J. Fish. Aquat. Sci., 37: 834-847

ODUM, E.P., 1971. Fundamentals of Ecology. 3rd edition. W.B. Saunders Co., Philadelphia, Pennsylvania.

ODUM, H.T, 1956. Primary production of flowing water. Lirnnol. Oceanogr., 1: 102-117.

ODUM, H.T. \& F.R. WILSON, 1962. Further studies on reaeration and metabolism of Texas bays, 1958-1960. Publ. Inst. Marine Sci. Univ. Tex., 8: 159-170.

OWENS, M., 1974. Methods for measuring production rates: Measurements on non-isolated natural communities in running waters. In: A manual on methods for measuring primary production in aquatic environments (R. A. VOLLENWEIDER. ed.): pp. 111-119. Blackwell Scientific Publications. Oxford.

PETERSEN, R.C. \& K.W. CUMMINS, 1974. Leaf processing in a woodland stream. Freshw. Biol., 4: 343-368.

ROSENFELD, J.S. \& R.J. MACKAY, 1987. Assesing the food base of stream ecosystems: alternatives to the $\mathrm{P} / \mathrm{R}$ ratio. Oikos, 50 (1): 141-147.

ROUNICK, J.S., M.J. WINTERBOURN \& G.L. LYON, 1982. Differential utilization of allochthonous and authochtonous inputs by aquatic invertebrates in some New Zealand streams: a stable carbon isotope study. Oikos, 39: 191-198.

ROUNICK, J.S. \& M.J. WINTERBOURN, 1986. Stable carbon isotopes and carbon flow in ecosystem.
BioScience, 36: 171-177.

RYDER, G.I. \& D. SCOTT, 1988. The aplicability of the river continuum concept to the New Zealand streams. Verh. Internat. Verein. Lirnnol., 23: 1441-1445.

SABATER, S., F. SABATER \& J. ARMENGOL, 1993. Ecología de los ríos mediterráneos. Investigación y Ciencia, 203: 72-79.

VANNOTE, R.L., G.W. MINSHALL, K.W. CUMMINS, J.R. SEDELL \& C.E. CUSHING, 1980. The River Continuum Concept. Can. J. Fish. Aquat. Sci., 37: 103137.

VIDAL-ABARCA, M.R., 1990. Los ríos de las cuencas áridas y semiáridas: una perspectiva ecológica comparativa y de síntesis. Scientia gerundensis, 16: 219-228.

VIDAL-ABARCA, M.R., C. MONTES, M.L. SUAREZ \& L. RAMIREZ-DIAZ, 1992a. An approach to the ecological characterization of arid and semiarid basins. GeoJournal, 26: 335-340.

VIDAL-ABARCA, M.R., M.L. SUAREZ \& L. RAMIREZ-DIAZ, 1992b. Ecology of Spanish semiarid streams. Limnética, 8: 151-160.

WINTERBOURN, M. J.; K. J. COLLIER. \& A. K. GRAESSER. 1988. Ecology of small streams on the west coast of the South Island, New Zealand. Verh. Internat. Verein. Lirnnol., 23: 1427-1431.

WINTERBOURN, M. J.; J. S. ROUNICK \& B. COWIE, 1981. Are New Zealand stream ecosystems really different? N. Z. J. Mar. Freshwat. Res., 15: 321-328. 
